\title{
Osteopontin Alleles Are Associated with Clinical Characteristics in Systemic Lupus Erythematosus
}

\author{
Tarak Trivedi, ${ }^{1}$ Beverly S. Franek, ${ }^{1}$ Stephanie L. Green, ${ }^{1}$ Silvia N. Kariuki, ${ }^{1}$ Marissa Kumabe, ${ }^{1}$ \\ Rachel A. Mikolaitis, ${ }^{2}$ Meenakshi Jolly, ${ }^{2}$ Tammy O. Utset, ${ }^{1}$ and Timothy B. Niewold ${ }^{1}$ \\ ${ }^{1}$ Section of Rheumatology and Gwen Knapp Center for Lupus and Immunology Research, University of Chicago,
5841 S. Maryland Avenue, MC0930, Chicago, IL 60637, USA
${ }^{2}$ Divison of Rheumatology and Rush Lupus Clinic, Rush University Medical Center, Chicago, IL 60612, USA
}

Correspondence should be addressed to Tammy O. Utset, tutset@medicine.bsd.uchicago.edu and Timothy B. Niewold, tniewold@medicine.bsd.uchicago.edu

Received 29 July 2011; Accepted 20 August 2011

Academic Editor: George Tsokos

Copyright (C) 2011 Tarak Trivedi et al. This is an open access article distributed under the Creative Commons Attribution License, which permits unrestricted use, distribution, and reproduction in any medium, provided the original work is properly cited.

\begin{abstract}
Variants of the osteopontin (OPN) gene have been associated with systemic lupus erythematosus (SLE) susceptibility and cytokine profiles in SLE patients. It is not known whether these alleles are associated with specific clinical phenotypes in SLE. We studied 252 well-characterized SLE patients from a multiethnic cohort, genotyping the rs11730582, rs28357094, rs6532040, and rs9138 SNPs in the OPN gene. Ancestry informative markers were used to control for genetic ancestry. The SLE-risk allele rs9138C in the 3' UTR region was associated with photosensitivity in lupus patients across all ancestral backgrounds (meta-analysis OR $=3.2,95 \%$ $\left.\mathrm{CI}=1.6-6.5, P=1.0 \times 10^{-3}\right)$. Additionally, the promoter variant rs11730582C demonstrated suggestive evidence for association with two hematologic traits: thrombocytopenia $(\mathrm{OR}=2.1, P=0.023)$ and hemolytic anemia $(\mathrm{OR}=2.6, P=0.036)$. These clinical associations with SNPs in the promoter and $3^{\prime}$ UTR regions align with previously reported SLE-susceptibility SNPs in OPN and suggest potential roles for these variants in antibody-mediated cytopenias and skin inflammation in SLE.
\end{abstract}

\section{Introduction}

Systemic lupus erythematosus (SLE) is an autoimmune disease characterized by the production of autoantibodies to nuclear antigens. The severity, prognosis, and manifestations of the disease are highly variable between patients. SLE commonly involves the hematopoietic, skin, musculoskeletal, and renal systems. Involvement of the respiratory, cardiovascular, and central nervous systems can also occur, and different patients typically exhibit different patterns of organ system involvement. Approximately ninety percent of cases are females, with the typical age of onset being in the reproductive years [1].

Genetic factors play a significant role in the pathogenesis of SLE. Familial studies have shown that 10-12\% of SLE patients have an affected first-degree relative, which is a twentyto fiftyfold increase in occurrence when compared to the general population [2]. Studies in monozygotic and dizygotic twins have shown that the disease co-occurs in monozygotic twins $24-69 \%$ of the time, while only $2-9 \%$ of the time in dizygotic twins [3]. While this data supports a strong genetic influence on SLE susceptibility, the varied concordance in different studies of monozygotic twins also implies a significant role for additional factors beyond genetic sequence variations. Genome-wide association and candidate gene studies have been successful in identifying a number of genes implicated in SLE susceptibility, including those for immunoglobulin receptors $(\mathrm{Fc} \gamma \mathrm{R})$ [4], and transcription factors such as STAT4 [5] and IRF5 [6, 7].

In order to discover mechanisms of SLE pathogenesis, candidate genes that produce cytokines are of interest [8]. Variants of the osteopontin (OPN or SPP1) gene have been associated with SLE susceptibility [9-11]. OPN is a secreted extracellular matrix cell adhesion glycoprotein. Its role in SLE may be explained through its diverse immunological functions, which include macrophage chemotaxis, T-Helper type 1 lymphocyte response, and B-cell activation [12]. High levels of OPN have been documented in biopsies of inflamed 
tissues in SLE and other autoimmune diseases [13, 14]. In addition, studies have shown that an increased plasma OPN level is correlated with increased SLE disease activity [15]. In mouse models, OPN is essential for the production of interferon-alpha (IFN- $\alpha)$ [16]. IFN- $\alpha$ levels are elevated in many SLE patients $[17,18]$, and high IFN- $\alpha$ is a heritable risk factor for SLE [19]. In previous work, we have shown that the SLE risk-associated allele of OPN rs9138C was associated with higher levels of serum OPN and IFN- $\alpha$ in young women and men with SLE [20]. This phenomenon in which a genetic risk factor for SLE impacts cytokine profiles has been demonstrated for a number of other SLE-risk loci as well [21-23].

There is some precedent for SLE-risk loci being associated with particular SLE clinical features [24-26] although much work remains to be done in this area. While OPN genetic variants have been linked to SLE susceptibility, little is currently known about whether OPN alleles are associated with particular clinical manifestations of SLE. One study demonstrated an association between lymphadenopathy and rs7687316 in the promoter region in European ancestry individuals [10]. Another study of 81 SLE patients of EuropeanAmerican ancestry demonstrated an association between a synonymous change in exon 7 with avascular necrosis and renal insufficiency [11]. We hypothesized that certain clinical subphenotypes of SLE would be associated with the presence of SLE risk alleles of the OPN gene, and that some of these associations would extend across different ancestral backgrounds. By identifying clinical traits associated with OPN alleles, we hoped to gain insight into both potential mechanisms of SLE pathogenesis and the diversity of clinical presentations between patients.

\section{Materials and Methods}

2.1. Patients and Samples. 252 patients clinically diagnosed with SLE were recruited from the outpatient rheumatology clinic at the University of Chicago and at Rush University, including 145 African-American, 67 European-American, 23 Hispanic-American, and 11 Asian-American patients. All patients fulfilled the American College of Rheumatology (ACR) Criteria score of 4 or higher [27]. The Institutional Review Boards at the respective institutions approved the study, and all subjects provided written informed consent. Table 1 contains demographic information on the patients included in the study.

2.2. Clinical Data. A comprehensive medical history relating to lupus and other immunologic illnesses was taken at the time of enrollment. ACR classification criteria for SLE were documented for each patient using patient history and laboratory testing. The "hematologic disease" ACR criterion is composite criteria composed which can be fulfilled by the presence of leukopenia, lymphopenia, thrombocytopenia, or hemolytic anemia. We chose to study each of these hematologic criteria separately, rather than studying the composite "hematologic disease" criterion. Similarly, the "immunologic disease" criterion is a composite of anti-Smith antibodies, anti-dsDNA antibodies, and antiphospholipid
TABLe 1: Demographic characteristics of the SLE population studied.

\begin{tabular}{cc}
\hline Self-reported ancestry & $N$ \\
\hline African American & 145 \\
European American & 67 \\
Hispanic American & 23 \\
Asian-American & 11 \\
\hline Age & yrs. \\
Range & $18-87$ \\
Mean & 44.38 \\
Std. deviation & 13.11 \\
\hline Gender & $n(\%)$ \\
Males & $21(8.33 \%)$ \\
Females & $231(91.67 \%)$ \\
\hline
\end{tabular}

antibodies, and we studied each of these individual components separately. Autoantibodies were assayed for all subjects in the University of Chicago Clinical Immunology Laboratory, using standard procedures and cutoff points for positive values. Anti-Sm and antiphospholipid antibodies were measured using ELISA, and anti-dsDNA was measured using the Crithidia luciliae method. Complete blood counts were done to assess blood cell subset counts. The prevalence of the various common clinical manifestations associated with SLE in our cohort is illustrated in Table 2.

2.3. Genotyping. We genotyped four haplotype-tagging and/ or SLE-associated SNPs within the OPN gene region. rs11730582 and rs28357094 are both in the $5^{\prime}$ promoter region of the OPN gene located on chromosome 4, and both have been previously associated with SLE. rs6532040 is in an intron and has not been previously associated with SLE susceptibility. rs9138 has been previously associated with SLE susceptibility and serum cytokines in SLE patients and is in the $3^{\prime}$ UTR region of osteopontin. Genotyping was performed using ABI Taqman assays-by-design primers for probes for each of the four SNPs on an ABI 7900HT PCR machine. Table 3 describes the genotype data at the four SNPs for patients in our study. All SNPs demonstrated the expected Hardy-Weinberg proportions when tested in each ancestral background individually (HW $P$ value $>0.01$ for all), supporting the validity of the genotype data.

2.4. Statistical Analysis. The genetic and clinical databases were merged, and STATA 8.2 was used for statistical analysis to determine associations between phenotypes and the four SNPs. The four SNPs chosen were not in high-linkage disequilibrium with each other $(r$-squared $<0.35$ for each pairwise SNP combination in each ancestral background), and thus there were no strong haplotypes formed across these 4 SNPs, and each SNP was analyzed separately. Because the frequency of the SNPs of interest and the frequency of SLE manifestations both vary by race, ancestry as a confounder had to be addressed in this study. To control this potential confounding, we first analyzed each self-reported ancestral background separately. Then, we used ancestry informative 
TABLE 2: Prevalence of clinical features in different ancestral backgrounds.

\begin{tabular}{|c|c|c|c|c|}
\hline Clinical feature & African Americans & European Americans & Hispanic Americans & Asian Americans \\
\hline Malar rash & 0.49 & 0.53 & 0.70 & 0.60 \\
\hline Discoid rash & 0.31 & 0.08 & 0.26 & 0.50 \\
\hline Photosensitivity & 0.08 & 0.08 & 0.13 & 0.30 \\
\hline Oral ulcers & 0.35 & 0.32 & 0.48 & 0.25 \\
\hline Arthritis & 0.85 & 0.86 & 0.81 & 1.00 \\
\hline Serositis & 0.34 & 0.35 & 0.43 & 0.20 \\
\hline Hemolytic anemia* & 0.10 & 0.05 & 0.20 & 0.25 \\
\hline Lymphopenia* & 0.83 & 0.76 & 1.00 & 0.75 \\
\hline Thrombocytopenia & 0.14 & 0.05 & 0.00 & 0.10 \\
\hline Leukopenia & 0.39 & 0.23 & 0.13 & 0.50 \\
\hline Neurologic symptoms & 0.14 & 0.12 & 0.09 & 0.00 \\
\hline Anti-Smith antibody & 0.41 & 0.18 & 0.39 & 0.40 \\
\hline Anti-DNA antibody & 0.51 & 0.56 & 0.22 & 0.60 \\
\hline Antiphospholipid antibody* & 0.42 & 0.43 & 1.00 & 0.25 \\
\hline ANA & 0.98 & 0.91 & 0.95 & 0.88 \\
\hline
\end{tabular}

* Data for these ACR criteria were only available for 130 SLE patients_-84 African Americans, 37 European Americans, 5 Hispanic Americans, and 4 Asian Americans.

TABLE 3: Minor allele frequencies across different ancestral backgrounds.

\begin{tabular}{lcccc}
\hline SNP & African Americans & European Americans & Hispanic Americans & Asian Americans \\
\hline rs11730582 C & 0.148 & 0.402 & 0.304 & 0.364 \\
rs28357094 G & 0.059 & 0.265 & 0.239 & 0.045 \\
rs6532040 G & 0.427 & 0.321 & 0.239 & 0.136 \\
rs9138 C & 0.164 & 0.265 & 0.250 & 0.500 \\
\hline
\end{tabular}

markers in a principal component analysis to generate quantitative values representing proportional ancestry. The full details of this analysis and graph of the first two principal components are shown in [28]. Principal components were used as a covariate in the logistic regression analyses to control for differences in proportional ancestry between SLE cases. In the case that a similar effect was observed across ancestral backgrounds, we performed a meta-analysis across groups using the same control for proportional ancestry between groups. Logistic regressions for categoricaldependent variables were performed in the STATA 8.2 program. Clinical manifestations were the dependent variables and were recorded as binary variables representing the presence or absence of each of the 11 ACR classification criteria. The four different OPN SNPs were used as the independent variables, and each SNP was modeled in an additive fashion. $P$ values shown in the paper are uncorrected for multiple comparisons. A $P$ value $<0.0031$ would withstand a Bonferroni correction for multiple comparisons, adjusting for the number of clinical variables tested in our study. Associations detected in this study which have a $P$ value of less than 0.05 but did not meet the threshold $P$ value of 0.0031 were described as "suggestive" or "possible" associations.

\section{Results}

3.1. rs9138 C Allele Is Associated with Photosensitivity. Photosensitivity was present in $8.64 \%(21 / 243)$ of patients in the cohort. As shown in Table 4, photosensitivity was strongly associated with the SLE risk allele rs9138 C across all ancestral backgrounds (meta-analysis $\mathrm{OR}=3.245, P=$ $0.001,95 \% \mathrm{CI}=1.609-6.542$ ). This association would withstand a Bonferroni correction for the number of phenotypes analyzed in this study. The prevalence of photosensitivity did not differ significantly between ancestral backgrounds (7.59\% in African Americans and 7.58 in European Americans, Table 2). Photosensitivity was not associated with the principal components representing genetic ancestry within each ancestral background, supporting the idea that differences in proportional ancestry or admixture were not confounding the analysis. Additionally, a similar pattern of association between rs9138 C and photosensitivity was observed in each ancestral background analyzed separately.

3.2. rs11730582 C Allele Is Associated with Thrombocytopenia and Hemolytic Anemia. The SLE risk-associated single nucleotide polymorphism rs11730582 C demonstrated a suggestive association with two different hematological manifestations (Table 4). Thrombocytopenia was associated with the rs11730582 $\mathrm{C}$ allele (meta-analysis $\mathrm{OR}=2.12, P=$ $0.023,95 \%$ CI $=1.11-4.04)$. Data for hemolytic anemia was available for 127 patients, as the samples from one of the sites did not assess this hematologic subcategory. A possible association between rs11730582 C and hemolytic anemia was observed in the patients in whom this data was available (meta-analysis $\mathrm{OR}=2.55, P=0.036,95 \% \mathrm{CI}=1.06-6.13$ ). 
TABLe 4: Associations between clinical features and OPN SNPs.

\begin{tabular}{|c|c|c|c|c|c|c|c|c|c|}
\hline \multirow{2}{*}{ SNP } & \multicolumn{3}{|c|}{ Photosensitivity } & \multicolumn{3}{|c|}{ Thrombocytopenia } & \multicolumn{3}{|c|}{ Hemolytic anemia } \\
\hline & OR & $95 \% \mathrm{CI}$ & $P$ value & OR & $95 \% \mathrm{CI}$ & $P$ value & OR & $95 \% \mathrm{CI}$ & $P$ value \\
\hline rs11730582 C & 1.74 & $0.90-3.39$ & 0.101 & 2.12 & $1.11-4.04$ & 0.023 & 2.55 & $1.06-6.13$ & 0.036 \\
\hline rs28357094 G & 0.99 & $0.42-2.39$ & 0.999 & 0.52 & $0.16-1.68$ & 0.273 & 0.26 & $0.03-2.05$ & 0.200 \\
\hline rs6532040 G & 0.59 & $0.28-1.22$ & 0.156 & 1.00 & $0.53-1.88$ & 0.999 & 1.31 & $0.53-3.22$ & 0.553 \\
\hline rs9138 C & 3.25 & $1.61-6.54$ & 0.001 & 1.58 & $0.79-3.13$ & 0.195 & 0.99 & $0.37-2.65$ & 0.977 \\
\hline
\end{tabular}

OR: odds ratio, $95 \%$ CI: $95 \%$ confidence interval of the odds ratio.

As might be expected, presence of thrombocytopenia and presence of hemolytic anemia were correlated with each other $(P=0.0013)$. In conditional analyses, each hematologic phenotype demonstrated similar suggestive evidence for association with rs $11730582 \mathrm{C}$ when conditioned on the other, and a higher odds ratio for association was observed for rs $11730582 \mathrm{C}$ in patients who exhibited both hematologic traits $(\mathrm{OR}=3.37, P=0.033)$.

\section{Discussion}

SLE is a clinically and genetically heterogeneous disease. It seems likely that the striking clinical heterogeneity is due at least in part to differences in the genetic determinants of disease between patients. We demonstrate an association between the most consistent SLE risk allele in the $3^{\prime}$ UTR region and photosensitivity and additionally show suggestive evidence that the promoter region of the gene is associated with hematologic phenotypes in SLE patients. This finding of two independent associations in the promoter and $3^{\prime}$ UTR regions has been observed previously with the OPN gene. In a case control study, this model of two independent associations was the best fit [10], and in our own previous work, we observed an antibody association with the promoter and a serum cytokine association with the $3^{\prime}$ UTR SNP rs9138 [20].

One limitation of this study is that a relatively small cohort with robust and detailed clinical data was analyzed. Thus, we were only able to detect associations with largeeffect sizes and cannot exclude any potential moderate strength associations that we did not detect in this study. Using a Bonferroni correction for multiple comparisons, the photosensitivity association with rs9138 C would remain significant, while the two possible hematologic associations with rs11730582 C would not. The genotype frequencies in our SLE cases were similar to those previously published in large-scale case-control experiments [9].

Interestingly, rs11730582 C was associated with two distinct hematological manifestations which share some aspects of pathogenesis: hemolytic anemia and thrombocytopenia. Previous studies have established that thrombocytopenia and hemolytic anemia in lupus typically have an autoantibody-mediated pathogenesis. $90 \%$ of lupus patients with thrombocytopenia have been shown to possess antibodies directed against either the glycoprotein IIb/IIIa or thrombopoietin receptor [29]. Hemolytic anemia in SLE is frequently a Coomb's test-positive, antibody-mediated hemolytic anemia. OPN in its secreted form functions as a cytokine involved in B-cell activation and plays a role in antibody production [14]. We have previously demonstrated the rs11730582 C allele was associated with an increased prevalence of antiribonuclear protein autoantibodies (antiRNP) [20]. These data in aggregate support a role for the promoter region in autoantibody formation in SLE.

Although the rs9138 C allele was not associated with hematological findings, it was strongly associated with photosensitivity in lupus patients. This association was particularly interesting given our previously published finding of the association of rs9138 C with increased levels of both osteopontin and IFN- $\alpha$ in SLE patients [20]. Numerous studies have indicated a central role of plasmacytoid dendritic cells (PDCs) and their secretion of IFN- $\alpha$ in SLE pathogenesis $[30,31]$. Additionally, OPN is essential for IFN- $\alpha$ production by murine PDCs [14]. It is known that cutaneous lupus is an autoimmune process characterized by photosensitivity, ultraviolet-light-induced apoptosis of keratinocytes, and an inflammatory infiltrate in superficial and deep compartments of the skin [30]. The histopathologic accumulation of PDCs and their subsequent secretion of IFN- $\alpha$ have been demonstrated in these photosensitive lesions [25, 32]. In light of these findings, the statistically significant association between the OPN gene variant rs9138 C and the clinical manifestation of photosensitivity may be supported by a cytokine-mediated pathogenic mechanism.

The rs11730582 C and rs9138 C alleles of the OPN gene have been previously and independently associated with increased risk of lupus. In this study, we find that both of these alleles are independently associated with distinct clinical features in SLE patients, supporting divergent roles for these alleles in the pathogenesis of SLE. This work will inform future genetic studies of the locus and provides intriguing hypotheses regarding the molecular pathogenesis of SLE which can be followed up in mechanistic studies.

\section{Funding Sources}

T. Trivedi received a Gina Finzi Memorial Summer Student Fellowship from the Lupus Foundation of America; T.O. Utset-Lupus Clinical Trials Consortium; T.B. NiewoldNIH R01 AR060861, NIH K08 AI083790, NIH P30 DK42086, NIAID Clinical Research Loan Repayment AI071651, NIH CTSA Core Subsidy Grant and CTSA Pilot Grants from UL1 RR024999, Lupus Research Institute Novel Research Grant, Alliance for Lupus Research Target Identification in Lupus Grant, and Arthritis National Research Foundation Eng Tan Scholar Award. 


\section{Conflict of Interests}

The authors report no financial conflict of interests.

\section{References}

[1] C. E. Weckerle and T. B. Niewold, "The unexplained female predominance of systemic lupus erythematosus: clues from genetic and cytokine studies," Clinical Reviews in Allergy and Immunology, vol. 40, no. 1, pp. 42-49, 2011.

[2] J. B. Harley, J. A. Kelly, and K. M. Kaufman, "Unraveling the genetics of systemic lupus erythematosus," Springer Seminars in Immunopathology, vol. 28, no. 2, pp. 119-130, 2006.

[3] D. Deapen, A. Escalante, L. Weinrib et al., "A revised estimate of twin concordance in systemic lupus erythematosus," Arthritis and Rheumatism, vol. 35, no. 3, pp. 311-318, 1992.

[4] J. E. Salmon, S. Millard, L. A. Schachter et al., "Fc $\gamma$ RIIA alleles are heritable risk factors for lupus nephritis in African Americans," Journal of Clinical Investigation, vol. 97, no. 5, pp. 1348-1354, 1996.

[5] E. F. Remmers, R. M. Plenge, A. T. Lee et al., "STAT4 and the risk of rheumatoid arthritis and systemic lupus erythematosus," The New England Journal of Medicine, vol. 357, no. 10, pp. 977-986, 2007.

[6] R. R. Graham, S. V. Kozyrev, E. C. Baechler et al., "A common haplotype of interferon regulatory factor 5 (IRF5) regulates splicing and expression and is associated with increased risk of systemic lupus erythematosus," Nature Genetics, vol. 38, no. 5, pp. 550-555, 2006.

[7] J. B. Harley, M. E. Alarcon-Riquelme, L. A. Criswell et al., "Genome-wide association scan in women with systemic lupus erythematosus identifies susceptibility variants in ITGAM, PXK, KIAA1542 and other loci," Nature Genetics, vol. 40, no. 2, pp. 204-210, 2008.

[8] S. N. Kariuki and T. B. Niewold, "Genetic regulation of serum cytokines in systemic lupus erythematosus," Translational Research, vol. 155, no. 3, pp. 109-117, 2010.

[9] S. Han, J. M. Guthridge, I. T. W. Harley et al., "Osteopontin and systemic lupus erythematosus association: a probable gene-gender interaction," PLoS One, vol. 3, no. 3, Article ID e1757, 2008.

[10] S. D’Alfonso, N. Barizzone, M. Giordano et al., "Two singlenucleotide polymorphisms in the $5^{\prime}$ and $3^{\prime}$ ends of the osteopontin gene contribute to susceptibility to systemic lupus erythematosus," Arthritis and Rheumatism, vol. 52, no. 2, pp. 539$547,2005$.

[11] A. C. Forton, M. A. Petri, D. Goldman, and K. E. Sullivan, "An osteopontin (SPP1) polymorphism is associated with systemic lupus erythematosus," Human Mutation, vol. 19, no. 4, p. 459, 2002.

[12] M. Scatena, L. Liaw, and C. M. Giachelli, "Osteopontin: a multifunctional molecule regulating chronic inflammation and vascular disease," Arteriosclerosis, Thrombosis, and Vascular Biology, vol. 27, no. 11, pp. 2302-2309, 2007.

[13] K. Masutani, M. Akahoshi, K. Tsuruya et al., "Predominance of Th1 immune response in diffuse proliferative lupus nephritis," Arthritis and Rheumatism, vol. 44, no. 9, pp. 2097-2106, 2001.

[14] D. Chabas, S. E. Baranzini, D. Mitchell et al., "The influence of the proinflammatory cytokine, osteopontin, on autoimmune demyelinating disease," Science, vol. 294, no. 5547, pp. 1731$1735,2001$.

[15] C. K. Wong, L. C. W. Lit, L. S. Tam, E. K. Li, and C. W. K. Lam, "Elevation of plasma osteopontin concentration is correlated with disease activity in patients with systemic lupus erythematosus," Rheumatology, vol. 44, no. 5, pp. 602-606, 2005.

[16] M. L. Shinohara, L. Lu, J. Bu et al., "Osteopontin expression is essential for interferon- $\alpha$ production by plasmacytoid dendritic cells," Nature Immunology, vol. 7, no. 5, pp. 498-506, 2006.

[17] C. E. Weckerle, B. S. Franek, J. A. Kelly et al., "Network analysis of associations between serum interferon-alpha activity, autoantibodies, and clinical features in systemic lupus erythematosus," Arthritis \& Rheumatism, vol. 63, no. 4, pp. 10441053, 2011.

[18] T. B. Niewold, D. N. Clark, R. Salloum, and B. D. Poole, "Interferon alpha in systemic lupus erythematosus," Journal of Biomedicine and Biotechnology, vol. 2010, Article ID 948364, 8 pages, 2010.

[19] T. B. Niewold, J. Hua, T. J. A. Lehman, J. B. Harley, and M. K. Crow, "High serum IFN- $\alpha$ activity is a heritable risk factor for systemic lupus erythematosus," Genes and Immunity, vol. 8, no. 6, pp. 492-502, 2007.

[20] S. N. Kariuki, J. G. Moore, K. A. Kirou, M. K. Crow, T. O. Utset, and T. B. Niewold, "Age- and gender-specific modulation of serum osteopontin and interferon- $\alpha$ by osteopontin genotype in systemic lupus erythematosus," Genes and Immunity, vol. 10, no. 5, pp. 487-494, 2009.

[21] R. Salloum and T. B. Niewold, "Interferon regulatory factors in human lupus pathogenesis," Translational Research, vol. 157, no. 6, pp. 326-331, 2011.

[22] T. Robinson, S. N. Kariuki, B. S. Franek et al., "Autoimmune disease risk variant of IFIH1 is associated with increased sensitivity to IFN-alpha and serologic autoimmunity in Lupus patients," Journal of Immunology, vol. 187, no. 3, pp. 12981303, 2011.

[23] S. N. Kariuki, B. S. Franek, A. A. Kumar et al., "Trait-stratified genome-wide association study identifies novel and diverse genetic associations with serologic and cytokine phenotypes in systemic lupus erythematosus," Arthritis Research \& Therapy, vol. 12, no. 4, p. R151, 2010.

[24] K. E. Taylor, E. F. Remmers, A. T. Lee et al., "Specificity of the STAT4 genetic association for severe disease manifestations of systemic lupus erythematosus," PLoS Genetics, vol. 4, no. 5, Article ID e1000084, 2008.

[25] I. T. W. Harley, T. B. Niewold, R. M. Stormont et al., "The role of genetic variation near interferon-kappa in systemic lupus erythematosus," Journal of Biomedicine and Biotechnology, vol. 2010, Article ID 706825, 11 pages, 2010.

[26] E. Sanchez, A. Nadig, B. C. Richardson et al., "Phenotypic associations of genetic susceptibility loci in systemic lupus erythematosus," Annals of the Rheumatic Diseases, vol. 70, no. 10, pp. 1752-1757, 2011.

[27] E. M. Tan, A. S. Cohen, J. F. Fries et al., "The 1982 revised criteria for the classification of systemic lupus erythematosus," Arthritis \& Rheumatism, vol. 25, no. 11, pp. 1271-1277, 1982.

[28] S. N. Kariuki, B. S. Franek, R. A. Mikolaitis et al., "Promoter variant of PIK3C3 is associated with autoimmunity against Ro and Sm epitopes in African-American lupus patients," Journal of Biomedicine and Biotechnology, vol. 2010, Article ID 826434, 2010.

[29] M. Kuwana, J. Kaburaki, Y. Okazaki, H. Miyazaki, and Y. Ikeda, "Two types of autoantibody-mediated thrombocytopenia in patients with systemic lupus erythematosus," Rheumatology, vol. 45, no. 7, pp. 851-854, 2006.

[30] L. Ronnblom, M. L. Eloranta, and G. V. Alm, "Role of natural interferon- $\alpha$ producing cells (plasmacytoid dendritic cells) in 
autoimmunity," Autoimmunity, vol. 36, no. 8, pp. 463-472, 2003.

[31] T. B. Niewold, "Interferon alpha as a primary pathogenic factor in human lupus," Journal of Interferon \& Cytokine Research. In press.

[32] L. Farkas, K. Beiske, F. Lund-Johansen, P. Brandtzaeg, and F. L. Jahnsen, "Plasmacytoid dendritic cells (natural interferon$\alpha / \beta$-producing cells) accumulate in cutaneous lupus erythematosus lesions," The American Journal of Pathology, vol. 159, no. 1, pp. 237-243, 2001. 


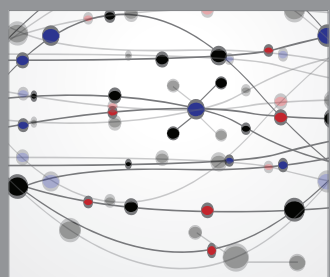

The Scientific World Journal
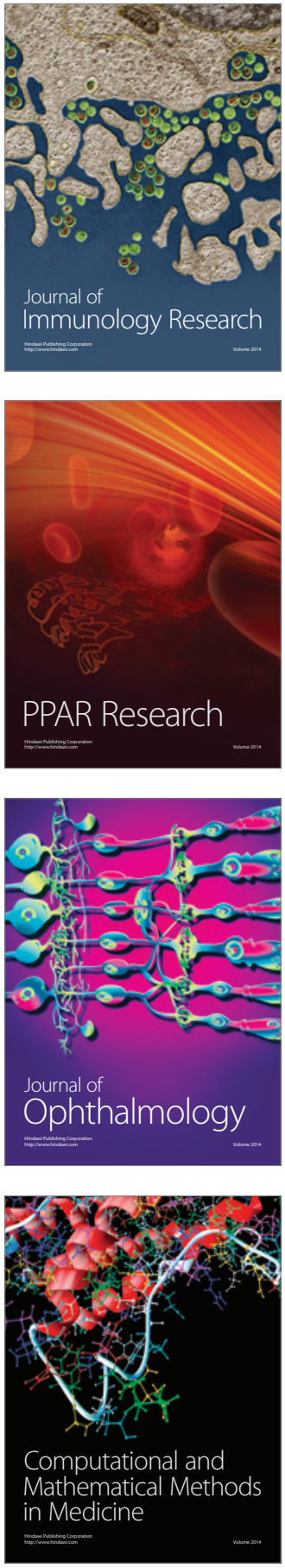

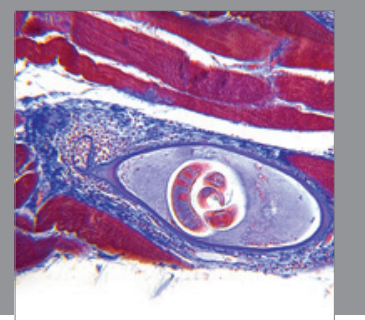

Gastroenterology

Research and Practice
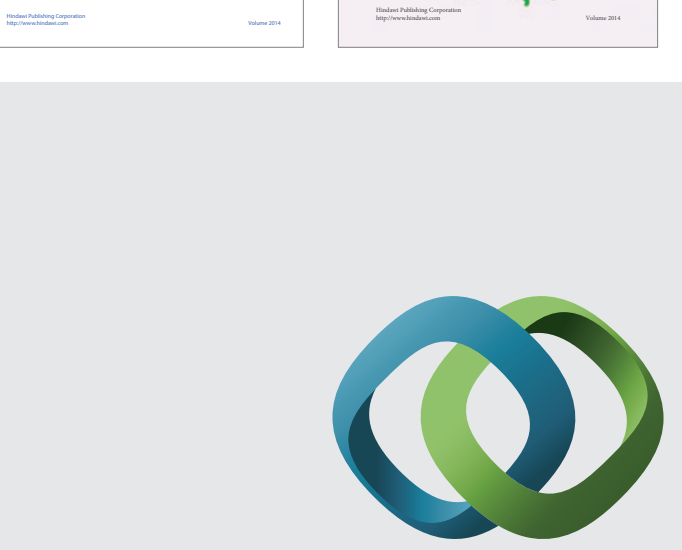

\section{Hindawi}

Submit your manuscripts at

http://www.hindawi.com
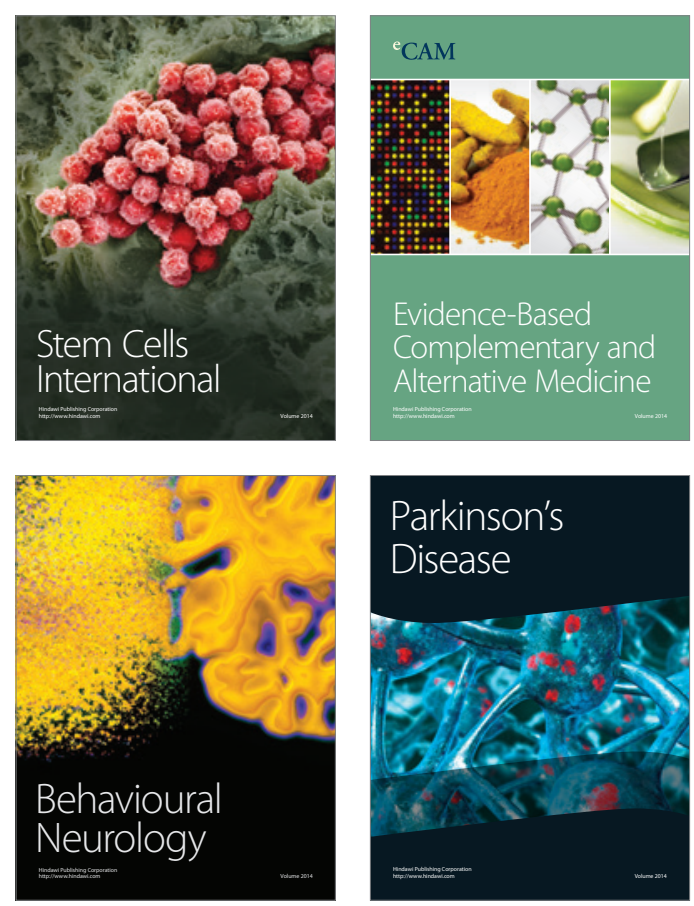

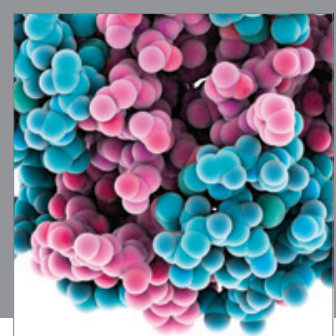

Journal of
Diabetes Research

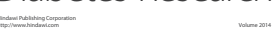

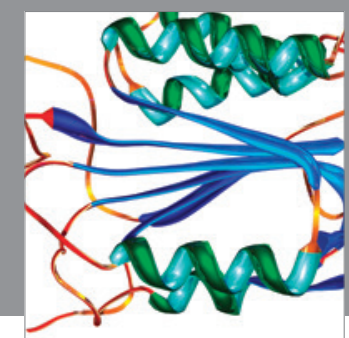

Disease Markers
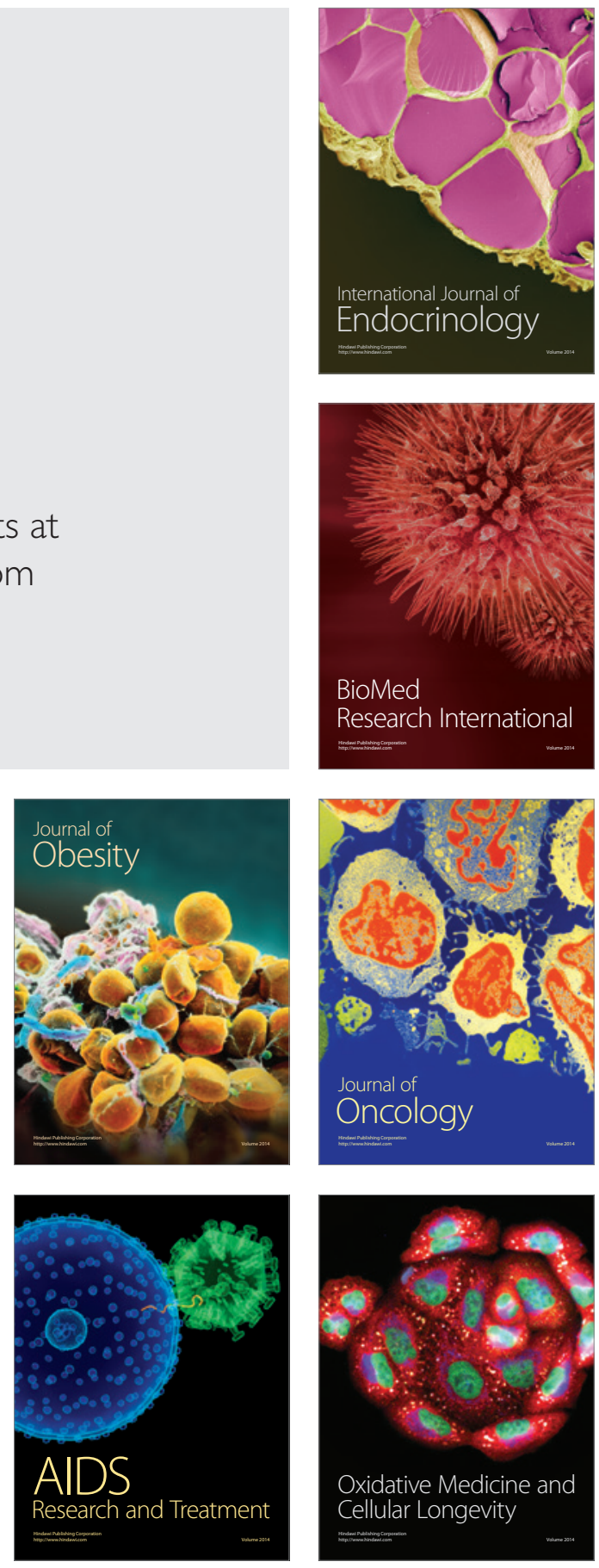